\title{
ReaR
}

Revista electrónica de AnestesiaR

Enero 2022

CASOS CLÍNICOS

\section{Sospecha mal y acertarás. Tuberculosis ósea como causa de dolor lumbar crónico. A propósito de un caso clínico.}

Correa Barrera JJ, Sánchez Solano S, Saz Castro R, San Juan Álvarez M.

Hospital Universitario Severo Ochoa. Leganés. Madrid

\section{Resumen}

A pesar de que la tuberculosis es considerada la segunda enfermedad infecciosa más letal a nivel mundial, actualmente en nuestro medio se trata de una enfermedad infrecuente. Las formas de tuberculosis extrapulmonar han experimentado un aumento relativo motivado, entre otras causas, por la inmigración procedente de zonas endémicas. Cuando la afectación tuberculosa es a nivel vertebral, el cuadro se manifiesta como un dolor lumbar crónico, que suele asociar síndromes larvados, poco específicos y que puede concluir en un cuadro óseo avanzado por retraso diagnóstico. El diagnóstico supone un desafío ya que requiere de una alta sospecha y de un abordaje multidisciplinar. El tratamiento en la actualidad es médico, considerándose la cirugía en contadas excepciones. El manejo de la tuberculosis vertebral supone un desafío para el anestesiólogo, que tendrá que abordar el manejo perioperatorio, la antibioterapia, así como el tratamiento del dolor mediante terapia farmacológica o de técnicas intervencionistas.

\section{Introducción}

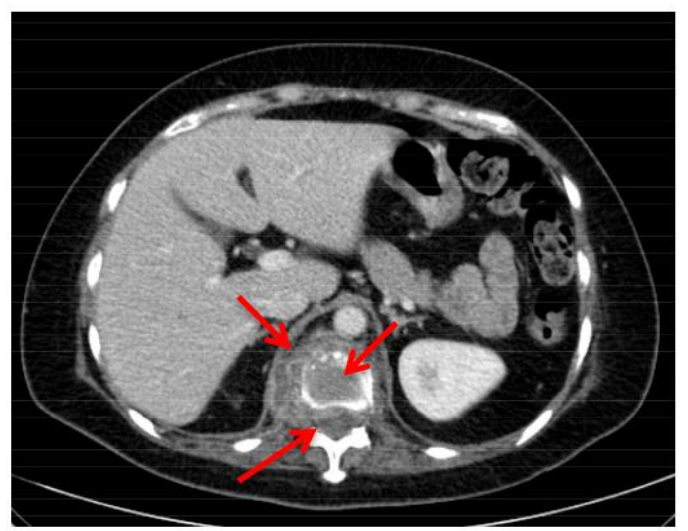

A pesar de que la tuberculosis es considerada la segunda enfermedad infecciosa más letal a nivel mundial, actualmente en nuestro medio se trata de una enfermedad infrecuente. Las formas de tuberculosis extrapulmonar han experimentado un aumento relativo motivado, entre otras causas, por la inmigración procedente de zonas endémicas. Cuando la afectación tuberculosa es a nivel vertebral, el cuadro se manifiesta como un dolor lumbar crónico, que suele asociar síndromes larvados, poco específicos y que puede concluir en un cuadro óseo avanzado por retraso diagnóstico. El diagnóstico supone un desafío ya que requiere de una alta sospecha y de un abordaje multidisciplinar. El tratamiento en la actualidad es médico, considerándose la cirugía en contadas excepciones. El manejo de la tuberculosis vertebral supone un desafío para el anestesiólogo, que tendrá que abordar el manejo perioperatorio, la antibioterapia, así como el tratamiento del dolor mediante terapia farmacológica 0 de técnicas intervencionistas.

La Tuberculosis (TB) es una enfermedad infrecuente en el mundo desarrollado. No obstante, a nivel mundial, continúa siendo unas de las enfermedades infecciosas más mortíferas, considerándose la segunda causa de muerte por enfermedad 
infecciosa después del Virus de inmunodeficiencia humana (VIH) (1).

La Tuberculosis extrapulmonar (TBEP), se define como aquella infección producida por Mycobacterium tuberculosis que afecta a tejidos $y$ órganos fuera del parénquima pulmonar. Representa el $20-25 \%$ de los pacientes con enfermedad tuberculosa (2). Aunque el número total de casos de TB ha disminuido, la reducción de TBEP ha sido menor, dando como resultado un incremento en el número de TBEP comparado con la Tuberculosis pulmonar (3).

La tuberculosis vertebral (TBV) es la afectación infecciosa de las estructuras óseas y/o articulares de la columna vertebral por el bacilo tuberculoso o bacilo de Koch. La TBV representa el $19 \%$ de los casos de infecciones vertebrales y $11 \%$ de las TBEP4. En los países desarrollados, la frecuencia de la Tuberculosis Osteoarticular (TO) es mayor en las etnias originarias de zonas endémicas tuberculosas en comparación con las personas nacidas en el país5.

Para el anestesiólogo, los casos de TBV, constituyen un desafío global, ya que pueden suponer una intervención en múltiples campos disciplinares que abarcan el manejo perioperatorio, la antibioterapia, así como el tratamiento del dolor mediante terapia farmacológica $o$ de técnicas intervencionistas.

\section{Descripción del caso}

Presentamos el caso de una mujer de 46 años de edad, natural de China, con antecedentes de hipertensión arterial (HTA) en tratamiento farmacológico, y con un cuadro larvado de aproximadamente un año de evolución de anemia con síndrome constitucional asociado, con episodios repetidos de diaforesis y sensación distérmica, sin identificar foco infeccioso.

Enmarcado en este cuadro la paciente presenta lumbalgia de 3 meses de evolución que asocia entumecimiento $\mathrm{y}$ debilidad, se valoró la intensidad del dolor mediante la Escala Visual Analógica (EVA), que puntúa de 0 a 10 tanto en reposo como en movimiento, siendo la puntuación de 3 con la paciente en reposo (dolor leve) y de 4 con el movimiento (dolor moderado).

Por otra parte se realizó una caracterización mediante la escala de dolor neuropático de 4 preguntas (DN4), donde una puntuación superior o igual a 4 indica un dolor sugerente de origen neuropático con alta sensibilidad y especificidad (Figura I). Nuestra paciente presentó hormigueo, quemazón $\mathrm{y}$ entumecimiento resultando una puntuación del NP4 de 3.

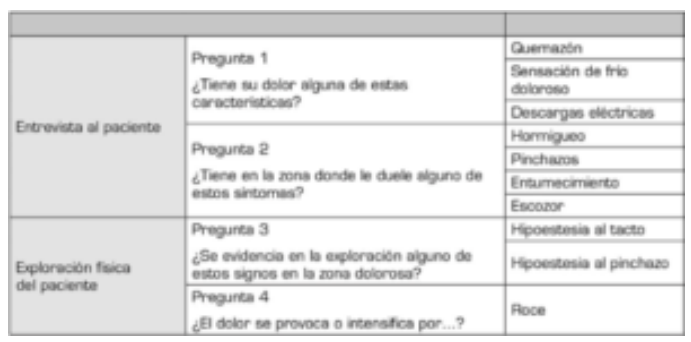

Figura I. Escala NP4 con cada pregunta y cada ítem. El valor de cada ítem positivo es de 1 . NP4 $\geq 4$ sugiere dolor de origen neuropático.

La paciente es valorada por parte de rehabilitación, con exploración a nivel de columna vertebral con apofisalgia -, DDS (Distancia dedo suelo) muy limitada, extensión conservada, rotaciones limitadas, talones puntillas sin claudicación y a nivel de MMII sensibilidad conservada sin alteraciones motoras. Se realiza Radiografía lumbar (Imagen I), que se informa como disminución del espacio intervertebral D12-L1. 

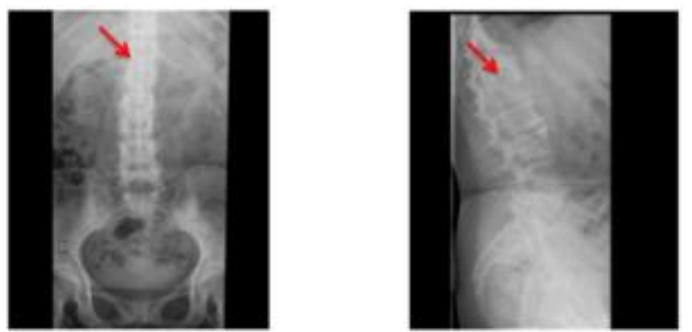

Imagen I: Radiografía lumbar frontal y lateral, con flechas señalando disminución del espacio D12-L1.

Se incluye a la paciente en la unidad de espalda, y se comienza con pauta de tratamiento analgésico con paracetamol, metamizol y tramadol junto con ejercicios de higiene postural y se amplía estudio con densitometría ósea (DO) (Imagen II). No se consideró la realización de técnicas intervencionistas por no disponerse de un diagnóstico de certeza.

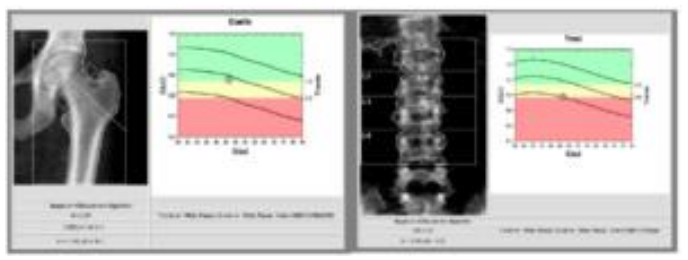

Imagen II: En la DO se aprecia osteopenia en columna lumbar y normalidad en cuello femoral.

A la espera de los resultados de la DO la paciente acude a urgencias por episodio de importante debilidad en miembros inferiores con dificultad para la marcha, encontrándose a la exploración apofisalgia en región lumbar y dorsal, paraparesia de miembros inferiores con fuerza proximal $4 / 5$ bilateral, distal $5 / 5$ bilateral, clonus aquíleo izquierdo agotable y no clonus aquileo derecho con babinsky extensor bilateral.

Ante la clínica sugerente de compresión de canal medular, se realiza estudio de imagen con TAC (Imagen III) y RMN (Imagen IV). Ambos estudios informan desestructuración centrada en nivel D10-D11, con destrucción de la vertiente articular de ambos platillos vertebrales. Ambos cuerpos vertebrales presentan aspecto expansivo y cambios edematosos óseos. El abombamiento del muro posterior condiciona marcada estenosis de conducto raquídeo con compresión medular y mielopatía compresiva. Los cambios se extienden también a los cuerpos vertebrales de D9 y D12. La evolución de los hallazgos sugiere proceso inflamatorio crónico como primera posibilidad diagnóstica. Ante estos hallazgos y la sospecha de una TBEP se solicita radiografía de tórax, sin hallazgos significativos $\mathrm{y}$ cultivo de esputo con resultado negativo para bacilos.
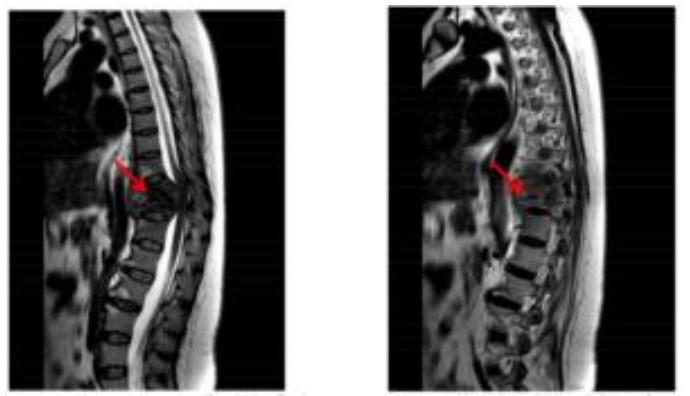

Imagen III: 2 cortes de RMN donde las flechas muestran compresión del cordón medular $\mathrm{y}$ afectación de los tejidos periféricos.

Se programa para toma de biopsias radioguiada en quirófano, y se remite muestra a laboratorio de anatomía patológica que informa de una tinción de Ziehl-Nielsen positiva y PAS positiva, apreciándose granulomas $\mathrm{y}$ células gigantes multinucleadas.

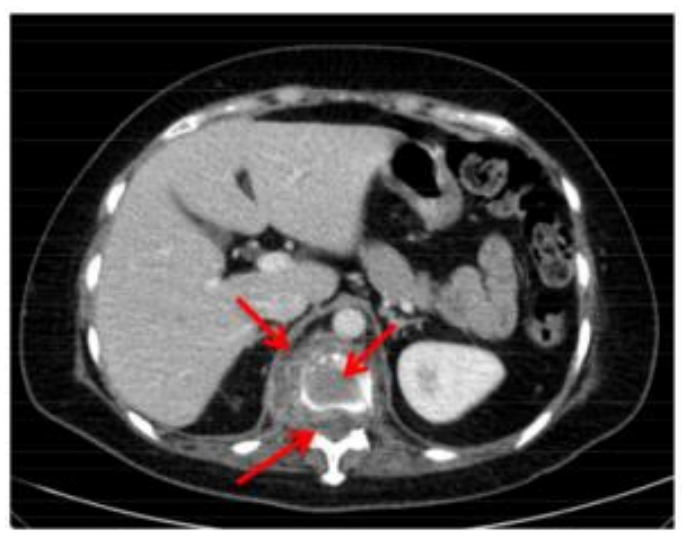

Imagen IV: Corte de en el que las flechas señalan afectación de tejidos, canal y cuerpo vertebral. 
La paciente se trató con analgesia durante el ingreso y recuperó la movilidad, fue dada de alta con tratamiento antituberculoso con 4 fármacos, rehabilitador $\mathrm{y}$ seguimiento trimestral con prueba de imagen mediante RMN.

\section{Discusión}

El dolor lumbar se define como un síndrome musculoesquelético o conjunto de síntomas siendo el principal la presencia de dolor focalizado en el segmento final de la columna vertebral (zona lumbar), en el área comprendida entre la costilla inferior y la región sacra, y que en ocasiones puede comprometer la región glútea, provocando disminución funcional6,7.

Se estima que entre un 60 y un $85 \%$ de la población sufrirá dolor lumbar en algún momento de su vida, de estos, tan solo el $15 \%$ tendrá un origen claro, y si bien en un gran porcentaje este cuadro remite de manera espontánea en menos de 6 semanas, en otros se requiere valorar aspectos psicológicos, sociológicos, culturales y laborales8.

El dolor lumbar crónico (DLC), es una entidad de manejo complejo, y en muchas ocasiones constituye un cuadro que requiere un abordaje multidisciplinar. Se trata de un término descriptivo que no implica connotaciones acerca del origen $\mathrm{o}$ fisiopatología de la enfermedad. Puede tratarse de una manifestación de diversas patologías (Tabla 1), con gravedad variable y con sustrato fisiopatológico muy diferente 7 .

La forma de abordar el manejo del DLC debe partir de la medicina basada en la evidencia. Transferir los hallazgos de la evidencia científica a la práctica clínica no es una tarea fácil, pero nos aporta ventajas importantes respecto a la práctica libre: un uso adecuado de los recursos, certeza del uso de terapias que dan resultado, y ante todo evitar vías de acción equivocadas o sin fundamento, que suponen un aumento del coste tanto económico como humano para el sistema sanitario $y$ el paciente, respectivamente9.

El DLC es el principal responsable de incapacidad y de absentismo laboral con el consiguiente elevado coste económico y deterioro en la calidad de vida de quienes lo padecen7.

Un tratamiento adecuado y orientado depende de un correcto diagnóstico diferencial, lo que a su vez supone conocer en profundidad la clasificación etiopatogénica del dolor lumbar crónico ( Tabla I).

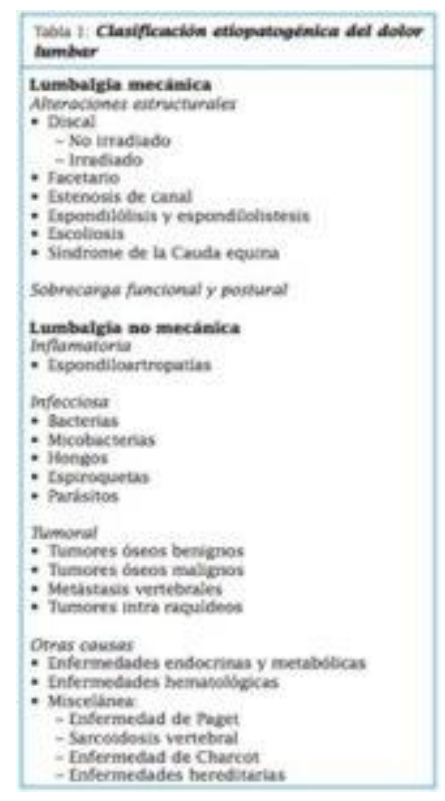

Tabla I: Clasificación etiopatogénica del dolor lumbar. Extraído de: Pérez-Torres, F., NuñezCornejo Piquer, C., Juliá-Mollá, C, BuadesSoriano, T., Ruíz-de la Torre, R., YbañezGarcía, D. y Muñoz-Mira, P. Capítulo 23: Lumbalgia. Reumatología. 403-419

Existen multitud de guías y tratados en la literatura que hacen revisiones profundas acerca del diagnóstico diferencial de esta patología. En nuestro medio la TBV constituye un cuadro de muy baja prevalencia dentro de las causas de dolor lumbar, y esto puede 
ocasionar un retraso diagnóstico de dicha enfermedad.

Para llegar a un adecuado diagnóstico de esta causa infrecuente de DLC, será necesario conocer en profundidad las manifestaciones clínicas características del cuadro de TBV.

La TBV suele iniciarse en la cara anterior de los cuerpos vertebrales y de allí extenderse al disco y a las vértebras adyacentes, pudiendo llegar, en los casos más severos, a invadir los tejidos blandos ocasionando abscesos paravertebrales, afectación del canal e incluso provocar compresión medular. En los pacientes jóvenes, con mayor frecuencia se comprometen las vértebras torácicas, mientras que en las personas ancianas la localización suele ser en vértebras de la región lumbar4.

La TBV suele cursar de forma insidiosa, con un lapso de tiempo prolongado entre el comienzo de los síntomas y el diagnóstico. Se manifiesta por dolor y/o tumefacción que se agrava de forma lentamente progresiva2,4,10. La alteración del estado general es inconstante y sería más frecuente en las formas multifocales, aunque en algunas ocasiones son asintomáticas2.

A pesar de que la radiografía estándar sigue siendo la prueba de primera elección, la RM se ha convertido en la prueba de referencia. La tomografía computarizada (TC) es indispensable si la RM está contraindicada o no está disponible, además con esta prueba de imagen se aprecian mejor las modificaciones de las estructuras óseas que con una radiografía, y es muy eficaz para mostrar los abscesos paravertebrales; además, puede detectar calcificaciones. Sin embargo, presenta una eficacia menor que la RM para identificar la afectación del disco intervertebral, las epiduritis y las compresiones neurológicas1.
El tratamiento adecuado de las infecciones tuberculosas de los huesos y las articulaciones es complejo. La selección de los fármacos antituberculosos y el control de la evolución de la enfermedad llevan implícito el abordaje multidisciplinar incluyendo a un especialista en enfermedades infecciosas5.

En las primeras décadas posteriores a la llegada de la terapia antituberculosa, el tratamiento estándar consistía en un régimen de tratamiento conjunto médico-quirúrgico. Con el paso del tiempo y dado los buenos resultados que se obtenían solo con tratamiento médico, se decidió ser más restrictivo en las indicaciones quirúrgicas 11.

Actualmente la intervención quirúrgica rara vez está indicada en el tratamiento inicial de la TB osteoarticular. Entre las posibles excepciones se encuentran los pacientes con enfermedad avanzada, progresiva o con cifosis vertebral de $40^{\circ}$ o más. La TB resistente a múltiples fármacos, también constituye una indicación relativa para el desbridamiento quirúrgico. Los pacientes con destrucción articular extensa e inmovilidad después de un ciclo adecuado de tratamiento médico son también posibles candidatos a cirugía5,11.

A pesar de que la incidencia de tuberculosis se considera muy baja en países desarrollados, son cada vez más los casos que se describen debidos a la inmigración desde zonas endémicas.

El elemento clave de la presentación del caso, radica en que en nuestra paciente, no se sospechó una posible complicación infecciosa poco frecuente, y se orientaron las pruebas diagnósticas hacia cuadros más comunes en nuestro medio, causa mecánica u osteoporótica, pasando por alto, datos clave, como pudieran ser el origen de la paciente ( 
China como zona endémica de tuberculosis) y el síndrome constitucional larvado asociado al dolor lumbar.

Actualmente las unidades de dolor crónico presentan una sobrecarga asistencial importante y un porcentaje importante de pacientes, acuden por DLC. Aunque la gran mayoría de casos corresponden a dolor lumbar mecánico, secundario a alteraciones estructurales, es importante tener presente causas menos comunes, especialmente en cuadros que no encajen en un diagnóstico concreto, o que asocien síndromes constitucionales.

Las imágenes del caso, la secuencia temporal, y las consecuencias derivadas, aportan un aprendizaje importante en la sospecha de enfermedades con baja incidencia en nuestro medio, y por tanto, una manera visual y didáctica de aprender a agudizar los sentidos en escenarios poco frecuentes.

Por todo esto, el caso clínico representa la importancia de una alta sospecha en el diagnóstico temprano del cuadro, para establecer un tratamiento adecuado y minimizar las consecuencias y costes asociados.

\section{Conclusiones}

El DLC constituye un cuadro clínico muy común que consume importantes recursos en el ámbito de la atención primaria y la consulta hospitalaria.

En muchas ocasiones el abordaje terapéutico es multidisciplinar, implicando a servicios de reumatología, rehabilitación, traumatología, neurocirugía y unidades del dolor.

Un correcto tratamiento pasa por un conocimiento profundo del cuadro y su diagnóstico diferencial.
Existen causas de dolor lumbar como la TBV cuya frecuencia en países desarrollados ha disminuido drásticamente en las últimas décadas, si bien han sufrido un repunte considerable con la inmigración.

Una adecuada sospecha clínica en pacientes procedentes de zonas endémicas permitirá establecer un tratamiento adecuado de manera precoz y minimizar la morbimortalidad asociada a la cuadro.

\section{Bibliografía}

1. World Health Organization: Drug-resistant TB: surveillance and response: supplement to global tuberculosis report 2014. (HTML)

2. Who report 2009. Global Tuberculosis control: epidemiología, strategy, financing. Geneva: World Health; 2009. (HTML)

3.Rieder HL, Snider Jr DE, Cauthen GM. Extrapulmonary tuberculosis in the United States. Am Rev Respir Dis. 1990;141:347-51. (HTML)

4. Colmenero JD, Jimenez-Mejias ME, Reguera JM, Palomino- Nicas J, Ruiz-Mesa JD, Marquez-Rivas J, et al. Tuberculous vertebral osteomyelitis in the new millenium: still a diagnostic and therapeutic challenge. Eur J Clin Microbiol Dis 2004;23: 477-83. (ㄴML)

5. Hayes AJ, Choksey M, Barnes N, Sparrow OC. Spinal tuberculosis in developed countries: difficulties in diagnosis. J RColl Surg Edinb 1996;41:192-6. (ㄴML)

6. Perez I, Alcorta I, Aguirre G. Guía de práctica clínica sobre la lumbalgia. Osakidetza. GPC 2007/1. Victoria-Gasteiz. ( Extraído del articulo guía de referencias). ( $\underline{\mathrm{PDF}}$ )

7. Casado Morales, M. ${ }^{a}$ Isabel, Moix Queraltó, Jenny, \& Vidal Fernández, Julia. (2008). Etiología, cronificación y tratamiento del dolor lumbar. Clínica y Salud, 19(3), 379-392. (

8. Pérez-Torres, F., Nuñez-Cornejo Piquer, C., Juliá-Mollá, C, Buades-Soriano, T., Ruíz-de la Torre, R., Ybañez-García, D. y Muñoz-Mira, P. Capítulo 23: Lumbalgia. Reumatología. 403419 
9. Ángel García, D., Martínez Nicolás, I., Saturno Hernández, P.J., \& López Soriano, F.. (2015). Abordaje clínico del dolor lumbar crónico: síntesis de recomendaciones basadas en la evidencia de las guías de práctica clínica existentes. Anales del Sistema Sanitario de Navarra, 38(1), 117-130. (medes)

10. Babhulkar S, Pande S. Extraspinal tuberculosis: unusual manifestations of osteoarticular tuberculosis. Clin Orthop 2002;398:114-20. (ㅍML)
11. García Ramos, R., Lado Lado, F.L., Túnez Bastida, V., Pérez Del Molino Bernal, M.L., \& Cabarcos Ortiz de Barrón, A. (2003).

Correspondencia al autor

Juan José Correa Barrera correabarrera83@gmail.com Facultativo Especialista de Area. Hospital Universitario Severo Ochoa. Leganés. Madrid

Aceptado para el blog en febrero de 2021 